\title{
On the challenges of drawing conclusions from $p$-values just below 0.05
}

Daniel Lakens

In recent years researchers have attempted to provide an indication of the prevalence of inflated Type 1 error rates by analyzing the distribution of $p$-values in the published literature. De Winter and Dodou (2015) analyzed the distribution (and its change over time) of a large number of $p$-values automatically extracted from abstracts in the scientific literature. They concluded there is a 'surge of $p$-values between 0.041-0.049 in recent decades' which 'suggests (but does not prove) questionable research practices have increased over the past 25 years'. I show the changes in the ratio of fractions of $p$-values between 0.041-0.049 over the years are better explained by assuming the average power has decreased over time. Furthermore, I propose that their observation that $p$-values just below 0.05 increase more strongly than $p$-values above 0.05 can be explained by an increase in publication bias (or the file drawer effect) over the years (cf. Fanelli, 2012; Pautasso, 2010), which has led to a relative decrease of 'marginally significant' $p$-values in abstracts in the literature (instead of an increase in $p$-values just below 0.05 ). I explain why researchers analyzing large numbers of $p$-values need to relate their assumptions to a model of $p$-value distributions that takes into account the average power of the performed studies, the ratio of true positives to false positives in the literature, the effects of publication bias, and the Type 1 error rate (and possible mechanisms through which it has inflated). Finally, I discuss why publication bias and underpowered studies might be a bigger problem for science than inflated Type 1 error rates, and explain the challenges when attempting to draw conclusions about inflated Type 1 error rates from a large heterogeneous set of $p$-values. 
1 RUNNING HEAD: PREVALENCE OF P-VALUES

2

3

4

5

6

On the challenges of drawing conclusions from $p$-values just below 0.05

8

9

10

Eindhoven University of Technology

11

12

Word Count: 6195

13

14

15

16

17

18

19

Author Note: I want to thank De Winter and Dodou for sharing their data, assisting in the re-analysis, and

reading an earlier version of this draft. All files required to reproduce the analyses in this article are available

21 from $\underline{\text { https://osf.io/ms } 4 \times 6 /}$

22

23

24 Correspondence can be addressed to Daniël Lakens, Human Technology Interaction Group, IPO 1.33, PO

25 Box 513, 5600 MB Eindhoven, The Netherlands. E-mail: D.Lakens@tue.nl.

26 


\section{Abstract}

In recent years researchers have attempted to provide an indication of the prevalence of inflated Type 1 error rates by analyzing the distribution of $p$-values in the published literature. De Winter and Dodou (2015) analyzed the distribution (and its change over time) of a large number of $p$-values automatically extracted from abstracts in the scientific literature. They concluded there is a 'surge of $p$-values between 0.041-0.049 in recent decades' which 'suggests (but does not prove) questionable research practices have increased over the past 25 years'. I show the changes in the ratio of fractions of $p$-values between $0.041-0.049$ over the years are better explained by assuming the average power has decreased over time. Furthermore, I propose that their observation that $p$-values just below 0.05 increase more strongly than $p$-values above 0.05 can be explained by an increase in publication bias (or the file drawer effect) over the years (cf. Fanelli, 2012; Pautasso, 2010), which has led to a relative decrease of 'marginally significant' $p$-values in abstracts in the literature (instead of an increase in $p$-values just below 0.05). I explain why researchers analyzing large numbers of $p$-values need to relate their assumptions to a model of $p$-value distributions that takes the average power of the performed studies, the ratio of true positives to false positives in the literature, the effects of publication bias, and the Type 1 error rate (and possible mechanisms through which it has inflated) into account. Finally, I discuss why publication bias and underpowered studies might be a bigger problem for science than inflated Type 1 error rates, and explain the challenges when attempting to draw conclusions about inflated Type 1 error rates from a large heterogeneous set of $p$-values. 
In recent years researchers have become more aware of how flexibility during the data-analysis can increase false positive results (e.g., Simmons, Nelson, \& Simonsohn, 2011). If the true Type 1 error rate is substantially inflated, for example because researchers analyze their data until a $p$-value smaller than 0.05 is observed, the robustness of scientific knowledge can substantially decrease. However, as Stroebe and Strack (2014, p. 60) have pointed out: 'Thus far, however, no solid data exist on the prevalence of such research practices'. Some researchers have attempted to provide an indication of the prevalence of inflated Type 1 error rates by analyzing the distribution of $p$-values in the published literature. The idea is that inflated Type 1 error rates lead to 'a peculiar prevalence of $p$-values just below 0.05 ' (Masicampo \& Lalande, 2012), the observation that '”just significant" results are on the rise' (Leggett, Loetscher, \& Jennions, 2015). researchers have drawn do not follow from the empirical data. The pattern of a peak of $p$-values just below $p=0.05$ observed by Leggett et al., (2013) does not replicate in other datasets of $p$-value distributions for the same journal in later years (Masicampo \& Lalande, 2012), in psychology in general (Hartgerink, van Assen, van Aert, Nuijten, b \& Wicherts, 2015; Kühberger, Fritz, \& Scherndl, 2014), or in scientific journals in general (De Winter \& Dodou, 2015). The peak in $p$-values observed in Masicampo \& Lalande (2012) is only surprising compared to an incorrectly modeled $p$-value distribution that ignores publication bias and its effect on the frequency of $p$-values above 0.05 (Lakens, 2014a, see also Vermeulen et al., 2015). The 'widespread' $p$-hacking observed by Head and colleagues (2015) disappears after controlling for a simple confound (Hartgerink, 2015). distributions and concluded that there is a 'surge of $p$-values between 0.041-0.049 in recent decades'. They improved upon earlier approaches to analyze $p$-value distributions by comparing the percentage of $p$-values over time (from 1990-2013). Two observations in the data they collected could seduce researchers to draw 
conclusions about a rise of $p$-values just below a significance level of 0.05 . The first observation the authors report is how from 1990 to $2013 p$-values between 0.041 and 0.049 rose more strongly than the percentage of $p$-values between $0.051-0.059$. The second observation is that the percentage of $p$-values that falls between 0.041-0.049 has increased more than the increase in the percentage of $p$-values between 0.001 $0.009,0.011-0.019,0.021-0.029$, and $0.031-0.039$ from 1990 to $2013^{1}$. The authors $(2015$, p. 37$)$ conclude that: "The fact that $p$-values just below 0.05 exhibited the fastest increase among all $p$-value ranges we searched for suggests (but does not prove) that questionable research practices have increased over the past 25 years."

I will explain why the data does not suggest an increase in 'questionable research practices'. First, I will discuss how the relatively stronger increase in $p$-values just below $p=0.05$ compared to $p$-values just above $p=0.05$ is not caused by a change over time in the percentage of $p$-values just below 0.05 , but by a change over time in the percentage of $p$-values above 0.05 . Perhaps surprisingly, $p$-values just above 0.05 increase much less than all other $p$-values. This might be due to a stricter interpretation of $p<0.05$ as support of a hypothesis, and less leniency for 'marginally significant' $p$-values just above this threshold. Second, I will explain why the relatively high increase in $p$-values between $0.041-0.049$ over the years can easily be accounted for by a decrease in the average power of studies. At the same time, I will illustrate why this increase in $p$-values just below 0.05 is unlikely to emerge due to an inflation of the Type 1 error rate due to optional stopping or trying out multiple analyses until $p<0.05$. I want to explicitly note that it was possible to provide these alternative interpretations of the data because De Winter \& Dodou (2015) shared all data and analysis scripts online. While I criticize their interpretation of data, I applaud their adherence to open science principles, which greatly facilitated cumulative science. Most importantly, the main point of this article is to highlight the challenges in drawing conclusions about inflated Type 1 error rates based solely on a large heterogeneous set of $p$-values.

As I have discussed before (Lakens, 2014a), it is essential to use a model of $p$-value distributions before drawing conclusions about the underlying reasons for specific distributions of $p$-values extracted from the scientific literature. A model of $p$-value distributions consists of four different factors. First, the 
$p$-value distribution depends on the number of studies where the null-hypothesis (H0) is true, and the number of studies where the alternative hypothesis (H1) is true. Second, the $p$-values for studies where H1 is true depend upon the power of the studies. Statistical power is the probability that a study yields a statistically significant effect, if there is a true effect to be found. Power is determined by the significance level, the sample size, and the effect size. Third, $p$-values for studies where H0 is true depend upon the Type 1 error rate chosen by the researcher (e.g., 0.05), and any possible mechanisms through which the Type 1 error rate is inflated beyond the nominal Type 1 error rate set by the researcher. When I talk about inflated Type 1 error rates in this article, I explicitly mean flexibility in dependent tests that are performed on the data (e.g., by performing a test after every few participants, flexibly deciding to exclude participants, or dropping or combining measurements) that have the goal to lead to a statistically significant result. When these statistical tests are dependent (e.g., analyzing the data after 20 participants, and analyzing the same data again after adding 10 additional participants) the Type 1 error rate inflation has a specific pattern where $p$-values between 0.041-0.049 become somewhat more likely than smaller $p$-values.

And finally, the $p$-value distribution in the published literature is influenced by publication bias. Publication bias is the tendency to publish statistically significant results (both because authors are more likely to submit those articles, as that editors and reviewers are more likely to evaluate such manuscripts more positively). The threshold at which $p$-values indicate a statistically significant result, as well as the leniency towards 'marginally significant' findings, both influence the frequency of observed $p$-values in the literature. It is important to look beyond simplistic comparisons between $p$-values just below 0.05 and $p$-values in other parts of the $p$-value distribution if the observed $p$-values are not explicitly related to a model consisting of the four factors that determine $p$-value distributions.

\section{Are $p$-values below 0.05 increasing, or $p$-values above 0.05 decreasing?}

De Winter and Dodou (2015) show there is a relatively stronger increase over time in $p$-values between 0.041-0.049 than in $p$-values between 0.051-0.059 (see for example their Figure 9). The data is clear, but the reason for this difference is not, and it is not explored by the authors. Although all $p$-values are increasing over time, the real question is whether $p$-values below $p=0.05$ are increasing more, or $p$ - 
124 values above $p=0.05$ are increasing less. A direct comparison is difficult, because a comparison across the $125 p=0.05$ boundary is influenced by publication bias. If publication bias increases, and less non-significant 126 results end up in the published literature due to the file-drawer problem, the percentage of papers reporting $127 p$-values smaller than 0.05 will also increase (even when there is no increase in $p$-hacking). Indeed, both 128 Pautasso (2010) as Fanelli (2012) have provided support for the idea that negative results have been 129 disappearing from the literature, which raises the possibility that the relative differences in $p$-values between 130 0.041-0.049 and 0.051-0.059 observed by De Winter and Dodou (2015) are actually caused by a relative 131 decrease in $p$-values between 0.051-0.059.

132 By comparing the relative differences between $p$-values between 0.031-0.039 and 0.041-0.049 over

133 the years on the one hand, and 0.051-0.059 and 0.061-0.069 on the other hand, we can examine whether

134 there is an increase in $p$-values between $0.041-0.049$ (due to an increase in the Type 1 error rate), or an 135 increase in publication bias (or the file-drawer problem), which leads to a lower percentage of $p$-values 136 between $0.051-0.059$. If there is an increase in the Type 1 error rate due to flexibility in the data analysis, 137 the biggest differences over time should be observed just below $p=0.05$ (in line with the idea of a surge of $138 p$-values between 0.041-0.049). However, there are reasons to assume the biggest difference will be observed in $p$-values just above $p=0.05$. As Lakens (2014a) noted, there seems to be some tolerance for $p$-values just above 0.05 to be published, as indicated by a higher prevalence of $p$-values between 0.051 0.059 than would be expected based on the power of statistical tests and an equal reduction of all $p$-values above 0.05 due to the file-drawer problem. If publication bias becomes more severe, we might expect a reduction in the tolerance for 'marginally significant' $p$-values just above 0.05 , and the largest changes in ratios should be observed above $p=0.05$. between $0.031-0.039$ to $p$-values between $0.041-0.049$ is pretty stable: $1.13,1.09$, and 1.11 , respectively. The ratio of $p$-values between $0.051-0.059$ to $p$-values between $0.061-0.069$ shows a surprisingly large reduction over the years: $2.27,1.94$, and 1.79 , respectively. It is important to note that flexibly analyzing 
150 above as below $p=0.05$. However, the ratio of $p$-values between 0.031-0.039 to $p$-values between 0.041 -

1510.049 should change much more than the ratio of $p$-values between $0.051-0.059$ to $p$-values between 0.061 -

1520.069 , because $p$-values are drawn from a relatively larger range above $p=0.05$, to a relatively small range

153 just below $p=0.05$. This surprisingly large change in ratios over time for $p$-values $0.051-0.059$ to 0.061 -

1540.069 indicates that instead of an increase in the Type 1 error rate of $p$-values below 0.05 , the real change

155 over time happens in the $p$-values between $0.051-0.059$.

156 The change over time in $p$-values just above $p=0.05$ might be explained by an increasingly strong 157 effect of the file-drawer problem. Where $p$-values between .051-0.059 (or 'marginally significant' results)

158 might have been more readily accepted as support for the alternative hypothesis in 1990-1997, $p$-values just

159 above 0.05 might no longer deemed strong enough support for the alternative hypothesis in 2005-2013.

160 This idea is speculative, but seems plausible given the increase in publication bias over the years (Fanelli,

161 2012; Pautasso, 2010), which suggests that non-significant results are less likely to be published in recent

162 years. It should be noted that $p$-values just above the 0.05 level are still more frequent than can be explained

163 just by the average power of the tests combined with publication bias that is equal for all $p$-values above

1640.05 (cf. Lakens, 2014a). In other words, this data is in line with the idea that publication bias is still slightly

165 less severe for $p$-values just above 0.05 , even though this benefit of $p$-values just above 0.05 has become 166 smaller over the years.

\section{How a changes in average power over the years affects ratios of $p$-values below 0.05}

The first part of the title of the article by De Winter and Dodou (2015), “A surge of $p$-values between $0.041-0.049$ " is based on the observation that the ratio of $p$-values between 0.041-0.049 increases more than the ratio of $p$-values between $0.031-0.039,0.021-0.029$, and $0.011-0.019$. There are no statistics reported to indicate whether these differences in ratios are actually statistically significant, nor are effect

172 sizes reported to indicate whether the differences are practically significant (or justify the term 'surge'), but 173 the ratios do increase as you move from bins of low $p$-values between 0.001-0.009 to bins of high $p$-values 174 between 0.041-0.049. 

there is a massive increase in the percentage of abstracts of papers in which $p$-values are reported over the years. As De Winter and Dodou (2015, p. 15) note: “In 1990, 0.019\% of papers (106 out of 563,023 papers) reported a p-value between 0.051 and 0.059 . This increased 3.6-fold to $0.067 \%(1,549$ out of 2,317,062 papers) in 2013. Positive results increased 10.3-fold in the same period: from 0.030\% (171 out of 563,023 papers) in 1990 to 0.314\% (7,266 out of 2,317,062 papers) in 2013.” De Winter and Dodou (2015) show $p$-values are finding their way into more and more abstracts, which points to a possible increase in the overreliance on null-hypothesis testing in empirical articles. This is an important contribution to the 183 literature.

The main question is how these differences in the ratios across the 5 bins below $p=0.05$ can be explained. De Winter and Dodou (2015) do not attempt to model their hypothesized mechanism by choosing values for the four factors of the model (the ratio of studies where $\mathrm{H} 0$ or $\mathrm{H} 1$ is true, the power of studies, the Type 1 error rate, and the presence of the file-drawer problem). However, this model contains all the factors that together completely determine the $p$-value distribution (except perhaps erroneously calculated $p$-values, which is also common, see Hartgerink et al., 2015; Vermeulen et al., 2015). Therefore, the hypothesis that flexibility in the data-analysis increases the Type 1 error rate must be translated into specific parameters for the factors in this model. It is only possible to explain the relative differences between the ratios of the different bins of $p$-values if we allow at least one of the parameters of the model to change over time. Because we are focusing on the $p$-values below 0.05 we can ignore the file drawer problem, assuming all disciplines that report $p$-values in abstracts use $\alpha=0.05$ (this is not true, but we can assume it applies to the majority of articles that are analyzed). The three remaining possibilities are a change in the average power of studies over time, a change in the inflated Type 1 error rate over time, and a change in the ratio of studies where $\mathrm{H} 0$ or $\mathrm{H} 1$ is true. I will discuss each of these three possible explanations in turn. 
power across the years. Remember that the distribution of $p$-values depends on the statistical power of the studies (or the average power of multiple subsets of studies, if heterogeneity in power is substantial), which is itself a function of the true effect size, the significance level, and the sample size (for formulas, see Cumming, 2008, Lakens \& Evers, 2014, for an online app, see http://rpsychologist.com/d3/pdist/). It is not difficult to model the ratios observed by De Winter and Dodou (2015) under the assumption that power decreases from 1990 to 2013. For example, if we assume the average power of studies was 55\% in 1990, and $42 \%$ in 2013, we will (given a large enough sample) observe the $p$-value distribution across the 5 bins as detailed in Table 1, with $29.86 \%$ of the $p$-values falling between 0.001 and 0.009 in 1990 , but only $19.93 \%$ of $p$-values falling between $0.001-0.009$ in 2013 . This is just the $p$-value distribution as a function of the power of the tests.

The total number of studies analyzed by De Winter and Dodou (2015) was 561516 in 1990, and in abstracts has increased by $10 \%$ over the years, and I chose $0.01 \%$ in 1990 and $0.1 \%$ in 2013 as the percentage of abstracts that report $p$-values (column 1 and 2 in Table 2). Assuming the average power was $55 \%$ in 1990 and $42 \%$ in 2013, we can calculate the expected number of observed $p$-values in 1990 and 2013 by simply multiplying the total number of articles (e.g., 561516) by the percentage of articles reporting $p$-values (e.g., 0.01), multiplied by the percentage of $p$-values expected in each $p$-value bin based on the assumed power (e.g., 0.300). The number of reconstructed $p$-values is presented in Table 2, columns 3 and (2015, Table 2, columns 5 and 6), indicating the chosen parameters for the model can reproduce the observed data.

Following De Winter and Dodou (2015), the fraction of the observed $p$-values in each of the five $p$-value bins can now be calculated by dividing the number $(\mathrm{N})$ of $p$-values in a specific bin for a specific

224 year (e.g., $\mathrm{N}=1676)$ by the total $(\mathrm{T})$ number of $p$-values (e.g., $\mathrm{T}=561516)$ to get the fractions for 1990 and 2015 (e.g., $1676 / 561516 \times 100=0.299)$. The reconstructed fractions (Table 3, column 1 and 2) are 
very similar to the observed fractions by De Winter and Dodou (columns 4 and 5). The main dependent variable De Winter and Dodou analyze is the fraction in 1990 divided by the fraction in 2013 (e.g., $0.299 / 1.993=6.674$, basically a ratio of fractions), and we can see the reconstructed ratios of fractions of 1990/2013 (column 3) closely resemble the observed ratios of fractions (column 6).

The reconstruction is close, but not perfect, for a number of reasons. First of all, there are very few data points from 1990, which will lead to substantial variation between expected and observed frequencies. For example, the reconstructed 1990/2013 ratio of fractions in the 0.021-0.029 bin (9.017) is smaller than the reconstructed ratio of fractions in the $0.031-0.039$ bin (9.417), but the pattern is reversed in the observed data (9.63 and 9.21, respectively). However, if we calculate the same ratios of fractions for 2013 with all other preceding years (e.g., 1991, 1992, 1993, etc.) we find a smaller ratio of fractions in the 0.021-0.029 bin than in the 0.031-0.039 bin for all the remaining 21 comparisons (see Table 4, columns 3 and 4). In other words, the model correctly predicts the ratios of fractions in 21 out of 22 comparisons between years, even when we chose the parameters for the model based on the 1990/2013 comparison. This provides strong support for the validity of the model that is used to reconstruct the ratios.

The model based on power differences similarly predicts that ratios for $p$-values between 0.031 0.039 should be very similar to those between $0.041-0.049$. The predicted $1990 / 2013$ ratio of fractions in the $0.031-0.039$ bin is 9.417 , and the predicted ratio of fractions for the $0.041-0.049$ bin is 9.740 , while the difference in observed ratios of fractions is much larger (9.21 and 11.28, respectively, see column 6 in Table 3). If the true difference is large (following the observed ratios) the ratio of fractions in the 0.041-0.049 bin should be consistently higher than the 0.031-0.039 bin across all years. If the reconstructed ratios are true, the difference between the two bins should be less pronounced across all year. When comparing 2013 to each of the 23 preceding years, the ratio of fractions in the 0.041-0.049 bin (see Table 4) is higher than for $p$-values in the $0.031-0.039$ bin in only 12 out of 23 comparisons ( $52 \%$ of the time). This can hardly be called a 'surge' of $p$-values between 0.041-0.049. This observation is not in line with the idea that the Type 1 error rate has increased, because an increase in Type 1 error rates due to flexibility in the data analysis 
typically assumes $p$-values between 0.041-0.049 increase more strongly than $p$-values between 0.031-0.039

252 (e.g., Head et al., 2015; Leggett et al., 2013; Masicampo \& Lalande, 2012).

Obviously a model that explains the observed $p$-value distribution only based on a change in the

254

255

256

257

258

259

260

261

262

263

264

265

266

267

268

269

270

271

272

273

274

275

average power of the studies (and sets the other factors to zero) is not likely to reflect the true state of affairs in the real world. Although we lack data about changes in the ratio of true to false effects examined over time (a worthwhile research question in itself), it seems reasonable to at least entertain the possibility of some changes over time for this factor of the model. For now, the most important conclusion is that a change in power over time can mathematically account for the observed changes in ratios of fractions in the different $p$-value bins. Moreover, the idea that power decreases over time is theoretically plausible, since such a decrease in power over time has been observed in some disciplines, such as psychology (Sedlmeier \& Gigerenzer, 1989), and the values of the parameters (55\% and $42 \%$ power) are plausible. At the same time, we can be certain power varies substantially across studies and research disciplines (e.g., Button et al., 2013; Sedlmeier \& Gigerenzer, 1989), and therefore the $p$-value distribution can be more accurately modeled by summing multiple $p$-value distributions across different research areas.

Changes in Type 1 error rates over time

Let's assume the average power has not changed over time, and instead try to reconstruct the observed data by De Winter and Dodou (2015) based on a change in the Type 1 error rates over time. The assumption is that there should be an increase in $p$-values just below $p=0.05$ because questionable research practices increase the number of false positives (De Winter \& Dodou, 2015, p. 6). A false positive or Type 1 error occurs when the null-hypothesis is true, but a statistically significant result is observed. When Type 1 error rates are not inflated (or nominal) $5 \%$ of the studies will observe $p$-values smaller than 0.05 . De Winter and Dodou focus on $p$-values between $0.041-0.049$ in the analysis of $p$-values with three digits (where $p$-values such as 0.040 will be absent because researchers are most likely to write 0.04 instead of $0.040)$, which means $0.8 \%$ of the time (0.049 minus 0.041$)$ a $p$-value within each of the five $p$-value bins will be observed ${ }^{2}$. By increasing the Type 1 error rate above $0.8 \%$, and doing so more strongly for higher 
276

277

278

279

280

281

282

283

284

285

286

287

288

289

290

291

292

293

294

295

296

297

298

299

300

301

$p$-value bins, we can attempt to reconstruct the consequences of an increase in false positives in the literature on the observed ratios of $p$-values over time.

The observed ratios of fractions by De Winter and Dodou (2015) show the 1990/2013 ratio is the smallest for $p$-values between 0.001-0.009 (i.e., 6.17), and substantially higher for $p$-values between 0.011 and 0.049 (see Table 3, last column). It is important to realize that the 1990/2013 ratios (see row 1, Table 4) with the large difference between the $0.031-0.039$ and $0.041-0.049$ bins ( 9.21 and 11.28 , respectively) is an outlier - the ratios in the two $p$-value bins are on average the same between 1992 and 2012. The pattern in the ratios for the 1990/2013 ratios can be reproduced based on inflated Type 1 error rates. However, reconstructing the ratios for the last twenty years requires an inflated Type 1 error rate that is unlikely to occur in real life.

One attempt to specify the parameters to model the ratios (but not the absolute values, because this proved to be too difficult solely based on an inflated Type 1 error rate) is presented in Table 5 (column 5). The ratio of studies where $\mathrm{H} 0$ is true to studies where $\mathrm{H} 1$ is true is set to 1 (a hypothesis is equally likely to be true or false), and the average power is assumed to be $57.5 \%$. The Type 1 error rate inflation over time has been increased based on a modest $p$-hacking strategy (collecting 50 participants in each condition, analyzing the data after every 10 participants until 100 have been collected). Although this would be substantial (it assumes $p$-hacking occurs in all studies where H0 is true), it is not impossible.

However, such a pattern of Type 1 error rates does not predict the ratio of fractions in the last 20 years, which were on average equal for the 0.031-0.039 and 0.041-0.049 bins. If we would change the Type 1 error rates to reconstruct the very similar ratios in the majority of the years in the $0.031-0.039$ and 0.041 0.049 bins only based on a change in Type 1 error rates, the Type 1 error rates should be equal across these bins, or even slightly lower in the $0.041-0.049$ bin compared to the $0.031-0.039 \mathrm{bin}$. This would be opposite to predictions based on flexibility during the data analysis (De Winter \& Dodou, 2015; Head et al., 2015; Leggett et al., 2013; Masicampo \& Lalande, 2012). The $p$-value distribution for true effects (based on the power of the studies) leads to a lower frequency of $p$-values in the $0.041-0.049$ bin than in the $0.031-0.039$ bin. A uniform inflation of the Type 1 error rate (e.g., increasing Type 1 error rates from 0.008 to 0.02 in 
302 all $p$-value bins) would add the same number of false positive $p$-values to each bin, but because the

303 frequency of true $p$-values decreases from bins with low $p$-values to bins with high $p$-values, the relative

304 increase is stronger in the $0.041-0.049$ bin than in the $0.031-0.039$ bin because the extra $p$-values from false

305 positives constitute a relatively larger increase. As Table 4 shows the ratios in the 0.031-0.039 bins and

306 0.041-0.049 bin are very similar in most of the years. Therefore, reconstructing these ratios would actually

307 require a lower increase in the Type 1 error rate in the $0.041-0.049$ bin than in the $0.031-0.039$ bin.

308 Researchers can (and probably do) $p$-hack studies where H1 is true. If such behavior increases over

309 time, we can expect the percentage of $p$-values from true effects in the 0.041-0.049 bin to increase in 2013

310 compared to 1990 . If we would incorporate this effect in the model, the relative increase over time in the

311 0.041-0.049 bin would be even stronger compared to the 0.031-0.039 bin. Again, we do not see huge

312 differences in the ratios between the 0.031-0.039 and 0.041-0.049 bins, which makes the explanation based

313 on flexibility in the data analysis even less likely.

314 Changes in the ratio of true to false effects that are examined

315 The third factor that could influence the ratios is the percentage of studies where true effects are 316 examined. A hypothesis is either true or not. The current analysis focuses on statistically significant findings

317 in the published literature (i.e., with $p$-values smaller than 0.05 ). This means that $p$-values are either true 318 positives (when $\mathrm{H} 1$ is true) or false positives (when $\mathrm{H} 0$ is true). The ratios calculated by De Winter and 319 Dodou (2015) can change over time based on the idea that for each published study in 2013 that examined 320 a true hypothesis, there is a much greater percentage of studies that examined a false hypothesis than in 321 1990. Most of the studies that examined a false hypothesis will end-up in the file drawer, but we can assume 322 (most of) the false positives end up in the literature. This greater number of false positives among the true 323 positives in 2013 compared to 1990 could, theoretically, explain the change in ratios over time.

324 However, the effect of a change over time of the ratio of true positives to false positives on the $p$ 325 value ratios in each of the five bins is really small. To account for the observed ratios by De Winter and 326 Dodou (2015), the changes in these ratios need to be quite substantial for a close reconstruction of the 327 observed data. For example, when keeping the power (i.e., $55 \%$ power) and Type 1 error rate (i.e., $0.08 \%$ 
328 in each $p$-value bin) constant over time, the observed ratios of fractions (although again, not the observed 329 absolute number of $p$-values) can be reconstructed purely based on a change in the $\mathrm{H} 0 / \mathrm{H} 1$ ratio over time

330 if we assume it was 10 times more likely to examine a true hypothesis compared to a false hypothesis in

331 1990, and at the same time assume it is 10 times more likely to examine a false hypothesis compared to a

332 true hypothesis in 2013 (for details, see the supplementary materials). That is a truly massive change over

333 time, where scientists are 100 times more likely to examine a false hypothesis than a true hypothesis in

3342013 compared to in 1990 . Without empirical data, it is not possible to conclusively reject this alterative

335 explanation, but it seems highly implausible. More subtle changes over time might exist, and it is

336 worthwhile to get data on the ratio of true to false hypotheses that researchers examine. However, with

337 respect to the current question, it is unlikely this factor of the model underlies the ratios observed by De

338 Winter and Dodou (2015).

339 To summarize, we can easily reconstruct the observed ratios by assuming a relatively small

340 decrease in power over the years (e.g., from 55\% to 42\%). On the other hand, while increases in Type 1

341 error rates can be used to reconstruct the observed ratios, the pattern of inflated Type 1 errors across the 5

342 bins of $p$-values is unlikely to emerge in real life, and the required difference in the ratio of true to false

343 hypotheses researchers examine is even less likely. Therefore, I conclude it is not very likely to be true that

344 there is a 'surge of $p$-values between 0.041-0.049', nor that these data suggest there is an increase in

345 questionable research practices over the last 25 years. A more plausible explanation is a small reduction in

346 the average power of experiments over the last twenty years. This could be caused by a stronger focus on

347 smaller effects over the years, after many larger effects have already been uncovered and researchers focus

348 more on moderators of known effects, or smaller novel effects.

\section{Discussion}

I hope to have illustrated the difficulty in accounting for the observed data by De Winter and Dodou

351 (2015) purely based on an increase in inflated Type 1 error rates over the years. The search for evidence of

352 an increase in questionable research practices in science in general, or at least across a large number of

353 studies, is starting to mirror the search for the ether. After repeatedly claiming to observe a rise in $p$-values 
354 just below 0.05 without providing substantial evidence for such a rise (De Winter \& Dodou, 2015; Head et 355 al., 2015; Leggett et al., 2013; Masicampo \& Lalande, 2012), and conflicting results across studies 356 examining this question, it is time that researchers investigating inflated Type 1 errors use a model of $p$ 357 value distributions to check their assumptions.

358 Any criticisms on the suggestion that changes in power over time are a more likely explanation of the observed ratios than inflated Type 1 error rates should propose different parameters for the model of $p$ value distributions. Regardless of any disagreements about the specific values for the parameters, the model of $p$-value distributions I have used here, based on the ratio of true positives to false positives, the power of studies, the Type 1 error rate, and the effect of publication bias, is a mathematical reality. If researchers aim to draw conclusions about the Type 1 error rate in the literature based on $p$-value distributions, they need to specify values for the parameters in this model, and interpret how plausible these values are. My explanation for the observed $p$-value distribution in De Winter and Dodou (2015) contains clearly testable predictions, such as the leniency of reviewers and editors to accept marginally significant $p$-values as support for a hypothesis, and the prediction that, on average, power has decreased from 1990-2013. Testing these predictions in future studies allows the values I have chosen for the parameters in the model to be either falsified or corroborated.

Analyzing huge numbers of $p$-values, which come from studies with large heterogeneity in effect sizes, might lack the sensitivity to provide support for an inflation of Type 1 error rates. Furthermore, automatically retrieving $p$-values from abstracts does not allow researchers to identify all theoretically relevant tests that are performed in an article that might contain multiple studies. Articles also contain many statistical tests not related to the main hypothesis, and might describe non-significant results without reporting statistics (e.g., no other differences reached statistical significance). A better approach seems to be to perform targeted analyses of small sets of similar studies, which might be able to yield support for $p$ hacking (e.g., Lakens, 2014b; Simonsohn et al., 2014; Van Assen et al., in press). I do not doubt that Type 
379

380

examine a hypothesis where the null hypothesis is true need to be large to observe an effect on the $p$-value distribution across a large number of studies.

\section{Conclusions}

Although it is important to control Type 1 error rates when performing statistical tests (e.g., Lakens, 2014c), I believe statistical power and publication bias due to the file drawer problem, and not $p$-hacking, are the biggest problems in the scientific literature. Without publication bias, Type 1 errors would be quite easily identified as they lead to follow-up research that will not observe the hypothesized effect. Even with publication bias, meta-analyses can identify sets of studies that lack evidential value, indicating the studies are a result of selection and reporting biases (Lakens, Hilgard, \& Staaks, in press; Simonsohn et al., 2014; Van Assen et al., in press). As the current analyses reveal, the $p$-value distributions in 1990 and 2013 in the data by De Winter and Dodou (2015) in 1990 and 2013 could be reproduced by assuming the average power of the studies was around $50 \%$. This suggests that from the outset only half of the performed studies could be expected to observe a statistically significant effect when the alternative hypothesis is true. This is clearly a huge waste of resources (especially in combination with publication bias). Inflated Type 1 errors and practices such as $p$-hacking have been very salient in recent years, but I believe it is worthwhile to point out that designing well-powered studies with high informational value (e.g., Lakens \& Evers, 2014) and preventing publication bias (e.g., Nosek \& Lakens, 2014) are at least, and I personally believe even more, important to improve our science. 


\section{Footnotes}

$398{ }^{1}$ The authors also analyze $p$-values with 2 digits (e.g., $p=0.04$ ), which reveal similar patterns, but here I

399 focus on the three digit data, which focuses on $p$-values between (for example) 0.041-0.049 because

400 trailing zeroes (e.g., $p=0.040$ ) are rarely reported).

$401{ }^{2}$ Because researchers round numbers to the nearest 3 digits, one might argue that instead of $0.8 \%$ of $p$ -

402 values between 0.041-0.049 when the null hypothesis is true, De Winter and Dodou (2015) actually focus

403 on $p$-values between $0.0405-0.0495$, which would mean $0.9 \%$ of the time a $p$-value within a single $p$ -

404 value bin will be observed. Using $0.8 \%$ or $0.9 \%$ Type 1 error rates in each bin does not influence the

405 reconstruction purely based on changes in power over time, and does not have consequences for any of

406 the conclusions based on the reconstructions.

407 
409

410

411

412

413

414

415

416

417

418

419

420

421

422

423

424

425

426

427

428

429

430

431

432

van Assen, M. A., van Aert, R., \& Wicherts, J. M. (in press). Meta-Analysis using effect size distributions of only statistically significant studies. Psychological Methods.

Button, K. S., Ioannidis, J. P., Mokrysz, C., Nosek, B. A., Flint, J., Robinson, E. S., \& Munafò, M. R. (2013). Power failure: why small sample size undermines the reliability of neuroscience. Nature Reviews Neuroscience, 14(5), 365-376.

Fanelli, D. (2012). Negative results are disappearing from most disciplines and countries. Scientometrics, 90, 891-904.

Hartgerink, C. (2015). Reanalyzing head et al. (2015): No widespread $p$-hacking after all? Retrieved from https://www.authorea.com/users/2013/articles/31568

Hartgerink, C. H. J., van Assen, M. A. M. L. M., van Aert, R. C. M., Nuijten, M. B., \& Wicherts, J. M. (2015). Distributions of $p$-values smaller than .05 in psychology: What is going on? Manuscript in Preparation.

Head, M. L., Holman, L., Lanfear, R., Kahn, A. T., and Jennions, M. D. (2015). The extent and consequences of $p$-hacking in science. PLOS Biology, 13:e1002106.

Kühberger, A., Fritz, A., \& Scherndl, T. (2014). Publication bias in psychology: A diagnosis based on the correlation between effect size and sample size. PLoS ONE 9(9): e105825. doi:10.1371/journal.pone.0105825

Leggett, N. C., Thomas, N. A., Loetscher, T., \& Nicholls, M. E. (2013). The life of p: "Just significant" results are on the rise. The Quarterly Journal of Experimental Psychology, 66, 2303-2309. doi: $10.1080 / 17470218.2013 .863371$

Lakens, D. (2014a). What $p$-hacking really looks like: A comment on Masicampo and Lalande (2012). The Quarterly Journal of Experimental Psychology, 68, 829-832.

Lakens, D. (2014b). Professors are not elderly: Evaluating the evidential value of two social priming effects through $p$-curve analyses. Available at SSRN: $\underline{\text { http://ssrn.com/abstract=2381936 }}$ 
Lakens, D. (2014c). Performing high-powered studies efficiently with sequential analyses. European Journal of Social Psychology, 44, 701-710. DOI: 10.1002/ejsp.2023.

Lakens, D., Hilgard, J., \& Staaks, J. (in press). On the reproducibility of meta-analyses: Six practical recommendations. BMC Psychology.

Lakens, D., \& Evers, E. R. (2014). Sailing from the seas of chaos into the corridor of stability: Practical recommendations to increase the informational value of studies. Perspectives on Psychological Science, 9(3), 278-292.

Masicampo, E. J., \& Lalande, D. R. (2012). A peculiar prevalence of $p$ values just below. 05. The Quarterly Journal of Experimental Psychology, 65(11), 2271-2279.

Nosek, B. A., \& Lakens, D. (2014). Registered reports. Social Psychology, 45(3), 137-141.

Pautasso, M. (2010). Worsening file-drawer problem in the abstracts of natural, medical and social science databases. Scientometrics, 85, 193-202.

Sedlmeier, P., \& Gigerenzer, G. (1989). Do studies of statistical power have an effect on the power of studies? Psychological Bulletin, 105(2), 309-316.

Simonsohn, U., Nelson, L. D., \& Simmons, J. P. (2014). P-curve and effect size: correcting for publication bias using only significant results. Perspectives on Psychological Science, 9(6), 666-681.

Simmons, J. P., Nelson, L. D., \& Simonsohn, U. (2011). False-positive psychology: Undisclosed flexibility in data collection and analysis allows presenting anything as significant. Psychological Science, 22, 1359-1366.

Stroebe, W., \& Strack, F. (2014). The alleged crisis and the illusion of exact replication. Perspectives on Psychological Science, 9, 59-71.

Vermeulen, I. E., Beukeboom, C. J., Batenburg, A. E., Stoyanov, D., Avramiea, A., Van de Velde, R. N., Oegema, D. (2015). Blinded by the light: $p$-value misreporting and excess $p$-values just below .05 in Communication Science. Under review with Communication Methods and Measures.

de Winter, J. C., \& Dodou, D. (2015). A surge of $p$-values between 0.041 and 0.049 in recent decades (but negative results are increasing rapidly too). PeerJ, 3, e733. 
Table $\mathbf{1}$ (on next page)

Expected percentage of $p$-values between $0.001-0.049$ based on $42 \%$ and $55 \%$ power. Note that columns do not sum to 0.55 and 0.42 because some $p$-values are not included in the analysis (e.g., $p$-values between 0.049-0.050). 


\begin{tabular}{|l|c|c|}
\hline & $\begin{array}{c}\text { Expected } p- \\
\text { values per bin } \\
\text { with 55\% power }\end{array}$ & $\begin{array}{c}\text { Expected } p \text { - } \\
\text { values per bin } \\
\text { with } 42 \% \text { power }\end{array}$ \\
\hline p0.001-p0.009 & 0.300 & 0.199 \\
\hline p0.011-p0.019 & 0.085 & 0.072 \\
\hline p0.021-p0.029 & 0.056 & 0.051 \\
\hline p0.031-p0.039 & 0.042 & 0.034 \\
\hline p0.041-p0.049 & 0.034 & 0.033 \\
\hline
\end{tabular}

2 
Table 2 (on next page)

Percentage of papers that report $p$-values in abstracts, and the number of reconstructed and observed (De Winter \& Dodou, 2015) p-values between 0.001-0.049 in 1990 and 2013 for each bin. 


\begin{tabular}{|l|c|c|c|c|c|c|}
\hline & $\begin{array}{c}\% p \text {-values in } \\
\text { abstracts 1990 }\end{array}$ & $\begin{array}{c}\% p \text {-values in } \\
\text { abstracts 2013 }\end{array}$ & $\begin{array}{c}\text { reconstructed \# } \\
p \text {-values 1990 }\end{array}$ & $\begin{array}{c}\text { reconstructed \# } p \text { - } \\
\text { values 2013 }\end{array}$ & $\begin{array}{c}\text { observed \# } \\
p \text {-values 1990 }\end{array}$ & $\begin{array}{c}\text { observed \# } \\
p \text {-values 2013 }\end{array}$ \\
\hline p0.001-p0.009 & 0.01 & 0.1 & 1676 & 46064 & 1770 & 44970 \\
\hline p0.011-p0.019 & 0.01 & 0.1 & 480 & 16690 & 462 & 14885 \\
\hline p0.021-p0.029 & 0.01 & 0.1 & 315 & 11698 & 268 & 10630 \\
\hline p0.031-p0.039 & 0.01 & 0.1 & 237 & 9189 & 240 & 9108 \\
\hline p0.041-p0.049 & 0.01 & 0.1 & 190 & 7629 & 178 & 8250 \\
\hline
\end{tabular}

2 


\section{Table 3(on next page)}

Ratio of fractions of reconstructed $p$-values and $p$-value ratios observed by De Winter and Dodou (2015) between 0.001-0.049 for 1990 and 2013.

$\mathrm{N}=$ number of $p$-values, $\mathrm{T}=$ total $p$-values 


\begin{tabular}{|l|c|c|c|c|c|c|}
\hline p-value bin & $\begin{array}{c}\text { reconstructed } \\
\text { fraction N/T } \\
1990\end{array}$ & $\begin{array}{c}\text { reconstructed } \\
\text { fraction N/T } \\
2013\end{array}$ & $\begin{array}{c}\text { reconstructed } \\
1990 / 2013 \text { Ratio } \\
\text { of fractions }\end{array}$ & $\begin{array}{c}\text { observed } \\
\text { fraction N/T } \\
1990\end{array}$ & $\begin{array}{c}\text { observed } \\
\text { fraction N/T } \\
2013\end{array}$ & $\begin{array}{c}\text { observed } \\
1990 / 2013 \text { ratio } \\
\text { of fraction }\end{array}$ \\
\hline $0.001-0.009$ & 0.299 & 1.993 & 6.674 & 0.315 & 1.945 & 6.17 \\
\hline $0.011-0.019$ & 0.085 & 0.722 & 8.454 & 0.082 & 0.644 & 7.83 \\
\hline $0.021-0.029$ & 0.056 & 0.506 & 9.017 & 0.048 & 0.460 & 9.63 \\
\hline $0.031-0.039$ & 0.042 & 0.398 & 9.417 & 0.043 & 0.394 & 9.21 \\
\hline $0.041-0.049$ & 0.034 & 0.330 & 9.740 & 0.032 & 0.367 & 11.28 \\
\hline
\end{tabular}

2 
Table 4 (on next page)

Ratios of fractions of the percentage of $p$-values in abstracts for 2013 relative to the percentages in 23 preceding years, for the $5 p$-value bins. 


\begin{tabular}{lccccc}
\hline Year compared & \multicolumn{5}{c}{ YEAR/2013 Ratio of fractions per $p$-value bin } \\
\cline { 2 - 6 } to 2013 & $0.001-0.009$ & $0.011-0.019$ & $0.021-0.029$ & $0.031-0.039$ & $0.041-0.049$ \\
\hline 1990 & 6.17 & 7.83 & 9.63 & 9.22 & 11.26 \\
1991 & 5.10 & 6.81 & 6.90 & 8.02 & 9.19 \\
1992 & 4.07 & 5.04 & 5.72 & 6.30 & 6.55 \\
1993 & 3.03 & 4.06 & 4.39 & 5.01 & 4.92 \\
1994 & 2.56 & 3.26 & 3.61 & 4.28 & 4.31 \\
1995 & 2.10 & 2.72 & 2.98 & 3.22 & 3.36 \\
1996 & 1.62 & 2.23 & 2.26 & 2.48 & 2.52 \\
1997 & 1.42 & 1.84 & 2.15 & 2.19 & 2.16 \\
1998 & 1.26 & 1.76 & 1.85 & 2.15 & 1.95 \\
1999 & 1.12 & 1.52 & 1.64 & 1.70 & 1.70 \\
2000 & 1.02 & 1.29 & 1.39 & 1.42 & 1.50 \\
2001 & 0.95 & 1.19 & 1.27 & 1.34 & 1.25 \\
2002 & 0.88 & 1.08 & 1.09 & 1.17 & 1.15 \\
2003 & 0.77 & 0.88 & 0.90 & 0.97 & 0.98 \\
2004 & 0.67 & 0.76 & 0.78 & 0.81 & 0.81 \\
2005 & 0.57 & 0.64 & 0.66 & 0.67 & 0.67 \\
2006 & 0.52 & 0.57 & 0.59 & 0.60 & 0.62 \\
2007 & 0.47 & 0.51 & 0.51 & 0.54 & 0.54 \\
2008 & 0.42 & 0.45 & 0.46 & 0.45 & 0.48 \\
2009 & 0.39 & 0.42 & 0.41 & 0.43 & 0.42 \\
2010 & 0.36 & 0.38 & 0.38 & 0.40 & 0.39 \\
2011 & 0.31 & 0.33 & 0.32 & 0.33 & 0.33 \\
2012 & 0.27 & 0.28 & 0.27 & 0.27 & 0.28 \\
\hline
\end{tabular}

2 
Table 5 (on next page)

Type 1 error rates, absolute number of reconstructed Type 1 errors between 0.0010.049 from 1990 to 2013 , and their ratio. 


\begin{tabular}{|l|c|c|c|c|c|}
\hline & $\begin{array}{c}\text { Type 1 error } \\
\text { rate 1990 }\end{array}$ & $\begin{array}{c}\text { Type 1 error } \\
\text { rate 2013 }\end{array}$ & $\begin{array}{c}\text { Significant } p \text {-values } \\
1990\end{array}$ & $\begin{array}{c}\text { Significant } p \text {-values } \\
2013\end{array}$ & $\begin{array}{c}\text { Reconstructed } \\
1990 / 2013 \text { ratio of } \\
\text { fractions }\end{array}$ \\
\hline p0.001-p0.009 & 0.008 & 0.009 & 1899 & 50338 & 6.44 \\
\hline p0.011-p0.019 & 0.008 & 0.014 & 581 & 17072 & 7.14 \\
\hline p0.021-p0.029 & 0.008 & 0.018 & 408 & 13706 & 8.16 \\
\hline p0.031-p0.039 & 0.008 & 0.022 & 327 & 12755 & 9.47 \\
\hline p0.041-p0.049 & 0.008 & 0.028 & 279 & 13262 & 11.56 \\
\hline
\end{tabular}

2 